\title{
Derivation of a Sample of Gamma-Ray Bursts from BATSE DISCLA Data
}

\author{
Maarten Schmidt \\ California Institute of Technology, Pasadena, California 91125
}

\begin{abstract}
We have searched for gamma-ray bursts (GRBs) in the BATSE DISCLA data over a time period of 5.9 years. We employ a trigger requiring an excess of at least $5 \sigma$ over background for at least two modules in the $50-300 \mathrm{keV}$ range. After excluding certain geographic locations of the satellite, we are left with 4485 triggers. Based on sky positions, we exclude triggers close to the sun, to Cyg X-1, to Nova Persei 1992 and the repeater SGR 1806-20, while these sources were active. We accept 1013 triggers that correspond to GRBs in the BATSE catalog, and after visual inspection of the time profiles classify 378 triggers as cosmic GRBs. We denote the 1391 GRBs so selected as the "BD2 sample". The BD2 sample effectively represents 2.003 years of full sky coverage for a rate of 694 GRBs per year. Euclidean $V / V_{\max }$ values have been derived through simulations in which each GRB is removed in distance until the detection algorithm does not produce a trigger. The BD2 sample produces a mean value $\left\langle V / V_{\max }\right\rangle=0.334 \pm 0.008$.
\end{abstract}

\section{INTRODUCTION}

We have been engaged for several years in an effort to derive a homogeneous sample of gamma-ray bursts (GRBs) from the continuous data stream transmitted by the Burst and Transient Source Experiment (BATSE). In particular, we have used the DISCLA data which provide the counts for each of the eight BATSE detectors in channels 1-4 on a time scale of $1024 \mathrm{~ms}$. The resulting BD2 sample of 1391 GRBs covers a time period of 5.9 years [3,4]. It includes 378 GRBs that are not in the BATSE catalog maintained on the BATSE web site. The BD2 sample has been used for a derivation of the characteristic luminosity and local space density of GRBs [4].

We will briefly review the procedures used to construct the BD2 sample, and analyze the differences between the BATSE catalog and the BD2 sample in some detail.

CP526, Gamma-Ray Bursts: $5^{\text {th }}$ Huntsville Symposium, edited by R. M. Kippen, et al. (C) 2000 American Institute of Physics 1-56396-947-5/00/\$17.00 


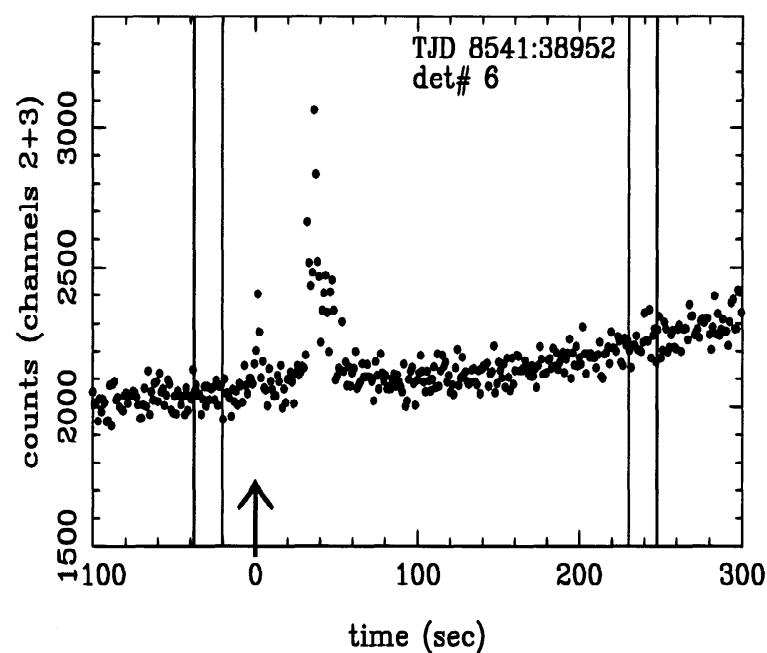

FIGURE 1. The test for the presence of a burst in time interval $0.000-1.024 \mathrm{~s}$ involves a linear interpolation between two $17.408 \mathrm{~s}$ averages of the background, one ending at time $-20.48 \mathrm{~s}$, the other starting at time $230.4 \mathrm{~s}$.

\section{DERIVATION OF THE BD2 SAMPLE}

For most of the time period covered by the BATSE catalog, the on-board trigger mechanism required that the counts in channels 2 and 3 covering the energy range $50-300 \mathrm{keV}$ exceed the background by $5.5 \sigma$ on a time scale of 64,256 or $1024 \mathrm{~ms}$ in two of the eight detectors. In our scarch based on DISCLA data, wc used channels $2+3$, and required an excess of $5 \sigma$ above background on a time scale of $1024 \mathrm{~ms}$ in two detectors.

The BATSE on-board trigger employs a background averaged over $17.408 \mathrm{~s}$ that is updated every $17.408 \mathrm{~s}$. We have taken advantage of the archival nature of the DISCLA data to use a background that is derived by linear interpolation from two averages taken over $17.408 \mathrm{~s}$, as shown in Figure 1. The interval of $20.48 \mathrm{~s}$ between the first background average and the test time is intended to alleviate the problem of detecting slowly rising GRBs [1].

We searched for defects in the DISCTA data, since these could lead to false triggers or affect the background. For the time period of TJD 8365-10528, we found around 151,000 defects, ranging from checksum errors that affected only one $1024 \mathrm{~ms}$ bin, to gaps caused by transmission problems, passage through the South Atlantic Anomaly, etc. Around each of these defects, we set up an exclusion time window such that the defect does not affect the background estimation.

The initial search yielded 7536 triggers. The geographic coordinates of the satellites at the time of trigger showed strong concentrations over W. Australia and over Mexico and Texas [3]. We then outlined geographic exclusion zones to avoid 


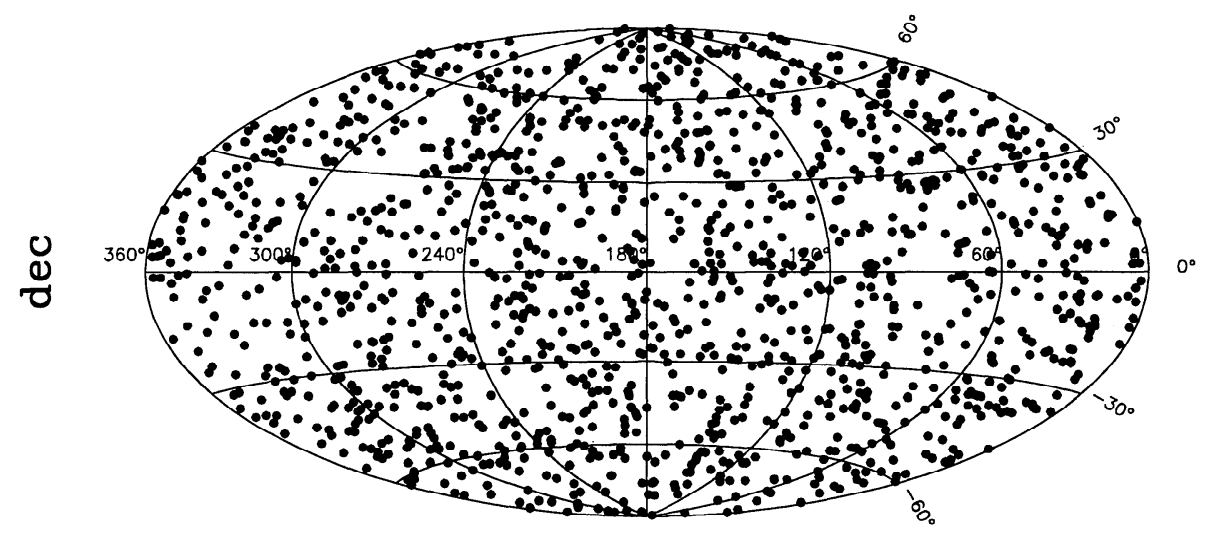

R.A.

FIGURE 2. Equatorial coordinates of $1391 \mathrm{GRB}$ in the BD2 sample

the trigger concentrations. With these exclusions in place, we were left with 4485 triggers. For each of these we derived celestial coordinates based on the relative response in all eight detectors and the orientation of the satellite, and other properties such as the duration, the hardness ratios, $V / V_{\max }$, etc.

The equatorial coordinates of these triggers showed clear concentrations that were identified as Cyg X-1, Nova Persei 1992, and solar flares along the ecliptic [3]. We excluded from consideration all triggers whose positions were within 23 deg of these sources while they were active. Most of the remaining triggers were either magnetospheric events or cosmic GRBs. For a more detailed description of the selection procedure, the reader is referred to [3]. We ended up with a sample of 1422 GRBs [3] which we call the BD1 sample.

Subsequently, we carefully investigated all our bursts that were either not in the BATSE catalog (for an example, see Fig. 3), or whose positions or times agreed poorly with the catalog data. We eventually rejected 31 of the sources in the BD1 sample (most of which were parts of long bursts or identified as the repeater SGR 1806-20), resulting in the BD2 sample of 1391 GRBs [4]. We show in Table 1 an updated accounting of the classification of all 4485 triggers.

The most important property among those derived for the GRBs in the BD2 sample is $V / V_{\max }$. Since redshifts are not know for most GRBs, $V / V_{\max }$ has to be evaluated in Euclidean space. It has usually been assumed that $V / V_{\max }=$ $\left(C_{\max } / C_{\min }\right)^{-3 / 2}$, where $C_{\min }$ is the minimum detectable burst signal, and $C_{\max }$ the maximum amplitude. Instead, we derive $V / V_{\max }$ of each GRB by carrying out a simulation in which we move the source out in Euclidean space, and at each step apply the detection algorithm to see whether the reduced burst is still detected [3]. In the process of moving out the source, it may get detected later 
TABLE 1. Classification of 4485 Triggers

\begin{tabular}{lrr}
\hline Description & reject & accept \\
\hline Within 23 deg of the sun when active & 963 & \\
Within 23 deg of CygX-1 when active & 827 & \\
Within 23 deg of Nova Persei when active & 418 & \\
Within 230.4 s of BA'TSE-listed burst & \multicolumn{3}{c}{1013} \\
Profile inspected: accepted as GRB & 378 \\
Profile inspected: rejected & 818 & \\
Part of a preceding long burst & 18 & \\
Soft repeater 1806-20 & 6 & \\
Near sun, soft spectrum & 44 & 1391 \\
\hline Total number of GRBs in the BD2 sample: & &
\end{tabular}

and later (depending on its time profile) and some burst signal may be included in the background. In some cases, the $C_{\max }$ part of the profile is never detected before the source disappears as it is being removed. Using $C_{\max }$ to derive $V / V_{\max }$ therefore tends to lead to an underestimate of $V / V_{\text {max }}$.

Using the procedure outlined above, the BD2 sample produces a mean value $\left\langle V / V_{\max }\right\rangle=0.334 \pm 0.008$ [4]. The deviation from the value 0.5 expected for a uniform space distribution reflects to first order the effect of using Euclidean space in its derivation rather than a relativistic cosmological model. Hence, the Euclidean $\left\langle V / V_{\max }\right\rangle$ is effectively a distance indicator, allowing derivation of the characteristic luminosity of GRBs [4].

\section{COMPARISON OF THE BATSE CATALOG AND THE BD2 SAMPLE}

Given that the BD2 sample was derived independently of the BATSE catalog, it is of interest to compare the two data sets.

1) The BD2 sample produces an all-sky rate of 694 per year, the BATSE catalog yields $\sim 690$ per year [2]. One might expect a larger rate in the BD2 sample since its limiting $\mathrm{S} / \mathrm{N}$ is 5.0, while for the BATSE catalog it is 5.5. However, the BD2 sample is limited to detections at the time scale $1024 \mathrm{~ms}$, while the BATSE catalog also includes time scales of 256 and $64 \mathrm{~ms}$.

2) The BD2 sample has 378 GRB not in the BATSE catalog, and the BATSE catalog has 130 GRB, detected at a time scale of $1024 \mathrm{~ms}$, that are not in the BD2 sample. These differences are related to the different depth of the two data sets ( $5 \sigma$ vs. $5.5 \sigma$ ), but they are also influenced by items (3)-(6) below.

3) The $\sim 151,000$ time exclusion windows used in the derivation of the BD2 sample are independent from those used in the BATSE search.

4) Following a BATSE trigger, the detection mechanism was disabled until data could be transmitted to the ground. After a BD2 detection, we disabled the software trigger for $230 \mathrm{~s}$. 


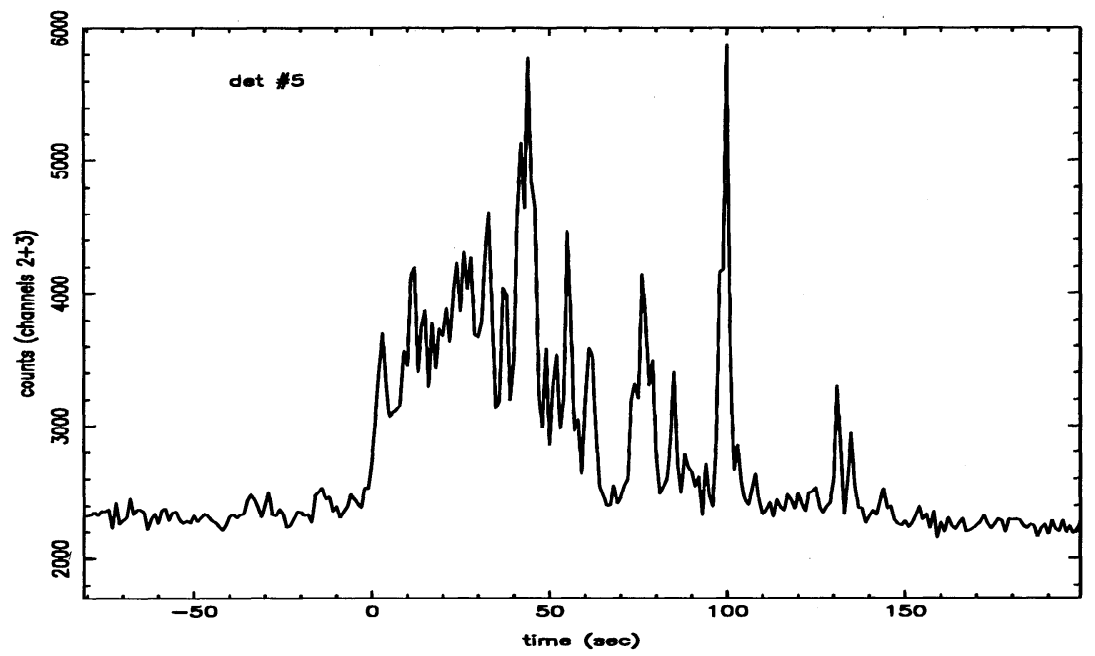

FIGURE 3. Example of a gamma-ray burst in the BD2 sample (TJD 8868:05731) that is not listed in BATSE catalog

5) The trigger criteria for the BATSE database were changed a number of times, see $[2]$.

6) In general, if the background stretches used in two searches are different, then if a source is detected precisely at the limiting $\mathrm{S} / \mathrm{N}$ in one of the searches, the probability that the other search finds the source is around $50 \%$. The backgrounds used in the BATSE search and the BD2 sample are independent when the BATSE background stretch is separated by less than $3 \mathrm{~s}$ from the time of detection and partly dependent when the separation is larger than $3 \mathrm{~s}$. Since we would find a substantial number of GRBs that are not in the BD2 by carrying out a scarch using different background stretches than those of Figure 1, we prefer to call the resulting collection of sources a sample. It should be stressed that the different samples so found are all statistically equivalent to each other, as long as one background choice is not to be preferred over the other.

\section{REFERENCES}

1. Higdon, J. C., and Lingenfelter, R. E., in Gamma-Ray Bursts, AIP Conference Proc. 384, New York: AIP, 1996, p. 402.

2. Meegan, C. A., et al. in Gamma-Ray Bursls, AIP Conference Proc. 428, New York: AIP, 1998, p. 3.

3. Schmidt, M., A\&A Suppl. 138, 409 (1999).

4. Schmidt, M., $\Lambda p J$ 523, L117 (1999). 